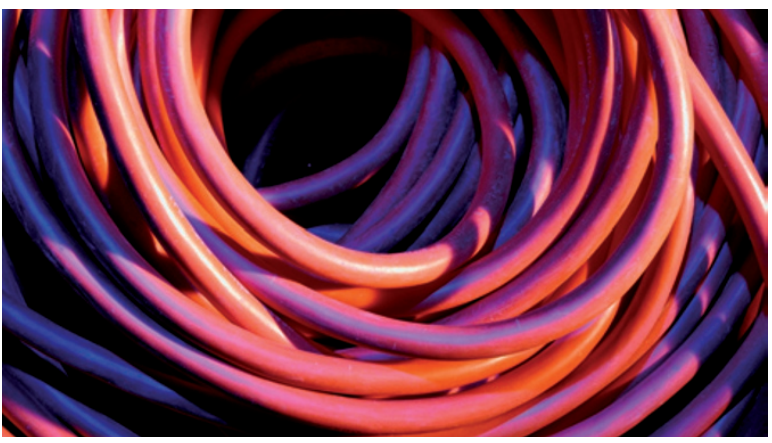

DEVELOPMENTAL BIOLOGY

\section{Inside tubes}

From cell-carrying blood vessels to the thin passageways of the lungs, the organs of our body harbour an assortment of variously sized tubes. A study now reports that the fundamental property of every biological tube - that of having a single cavity, or lumen - is genetically controlled.

Michel Bagnat and colleagues focused on the largest tube in the body, the gut. A mutation in the zebrafish tcf2 (transcription factor 2, hepatic) gene causes the gut to have not one, but many lumens. The wild-type gut normally develops through the fusion of more than one adjacent lumen, and so the authors suggest that tcf2 mutations interrupt the normal coalescence process. But how does tcf 2 mediate its function? A DNA microarray experiment pulled out a candidate effector, claudin15 (cldn15): cldn15 transcripts are absent from the gut of $t c f 2^{2169}$ mutants, and knocking down cldn15 has the same consequence as tcf2 inactivation.

Claudins form pores that mediate the movement of ions across an epithelium. cldn15 could therefore regulate the passage of ions across the developing gut wall, and the ensuing influx of fluid into the tube cavity might somehow regulate lumen coalescence. This hypothesis was supported in an in vitro lumen assay: when ion-impermeable cells were made to express cldn15, they became 'leaky' to ions, and the lumen of many cysts expanded. Similarly, treating cells with compounds that favour the accumulation of fluid in the lumen led to the formation of a single larger lumen, which was generated by a process that mimics the lumen coalescence that occurs during development. Finally, the authors showed that disruption of electrochemical gradient formation in vivo produced a phenotype that was similar to that of tcf $2^{2169}$ mutants.

Tube development is often associated with changes in the adhesive properties of cells. Although these more conventional mechanisms might also be operating here, this work points firmly to the involvement of an electrochemical gradient and the physical pressure that is exerted by an influx of fluid: the latter would promote both tube expansion and generate the force required to fuse adjacent lumens.

Tanita Casci

ORIGINAL RESEARCH PAPER Bagnat, M. et al. Genetic control of single lumen formation in the zebrafish gut. Nature Cell Biol. 15 July 2007 (doi:10.1038/ncb1621) WEB SITE

Didier Stainier's laboratory: http://www.ucsf.edu/dyrslab

TECHNOLOGY

\title{
(Truly) on and off at the flick of a switch
}

Many systems have been generated to turn gene expression on and off in an inducible way, but so far each has shown shortcomings such as leakiness or irreversibility. Now, by coupling RNAi to an inducible system that utilizes repressor proteins, James Collins and colleagues have obtained a genetic switch that guarantees tight and tunable control of gene expression.

The authors engineered a synthetic gene network (LTRi) involving the bacterial LacI and TetR transcriptional repressors and an RNAi component coding for a shorthairpin RNA (shRNA). The target of the shRNA is a complementary $19-b p$ sequence placed in the $3^{\prime}$ UTR of the gene of interest.

The default state of the switch is off: here, LacI represses the transcription of the gene of interest and also of the TetR repressor so that TetR cannot suppress shRNA transcription. Should any leakage occur, the shRNA molecule further knocks down the gene, post-transcriptionally. The addition of isopropyl- $\beta$-thiogalactopyranoside (IPTG), which inhibits LacI repressor function, turns the gene on by releasing its LacImediated repression and also LacI-mediated repression of TetR, which can then suppress transcription of the shRNA.

Given the modular structure of the switch, the authors were able to test each module independently; this was done by transiently transfecting human cells, using EGFP as the gene of interest. Adding the RNAi module or the LacI-repressor module alone achieved an $\sim 80 \%$ or $\sim 85 \%$ reduction of EGFP expression, respectively; by contrast, combining the modules altogether attained a greater than 99\% repression of the transgene.

Stable transfections of LTRi-EGFP in different human cell lines showed that, in each cell type, the switch can be repeatedly and reversibly flipped on and off by IPTG addition or removal, and gene expression levels can be finely tuned by using intermediate concentrations of IPTG.

The authors confirmed the tightness of the switch by showing that it can completely suppress the expression of the highly toxic $\alpha$-chain of diphtheria toxin - of which just a molecule suffices to kill a cell - and of the cre recombinase in primary mouse fibroblasts. Moreover, the tunable property of the switch makes it a unique tool to study phenotypes that depend on threshold levels of gene expression, as the authors showed for BAX-driven apoptosis of primary mouse fibroblasts.

Spanning from basic research to disease study and gene therapy, the potential applications of this tunable switch seem to be immense.

Francesca Pentimalli

ORIGINAL RESEARCH PAPER Deans, T. L., Cantor, C. R. \& Collins, J. J. A tunable genetic switch based on RNAi and repressor proteins for regulating gene expression in mammalian cells. Cell 130, 363-372 (2007)

FURTHER READING Benner, S. A. \& Sismour, J. J. Synthetic biology. Nature Rev. Genet. 6, 533-543 (2005)

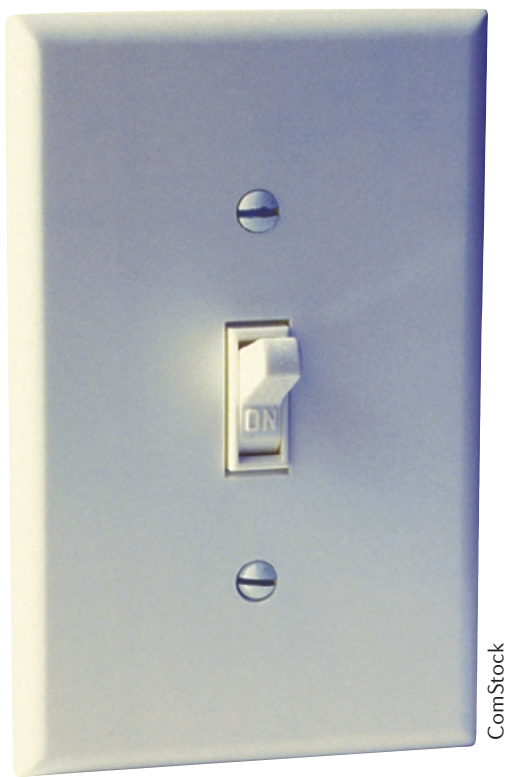

